\title{
A Statistical Model for an Automatic Procedure to Compress a Word Transcription Dictionary
}

\author{
Fériel MOURIA-BEJI (Member IEEE) \\ ENSI/LIA. Artificial Intelligence Group. \\ BP. 275, Cité Mahrajène, 1082 Tunis, Tunisia \\ E-mail : Mouria-Beji<maddouri@ensi.rnrt.tn>
}

\begin{abstract}
Various experiments have conclusively shown that superior continuous speech recognition performance is obtained when using context-dependent phonemic models. However, we have observed that using an explicit context-dependent phonemic model can yield many transcriptions for a single lexicon entry. In this work, we study the compression of the word transcription dictionaries (WTD) into a more compact form to balance the need between flexibility and reliability. Based on a measure of a likelihood function, a statistical model for an automatic procedure to compress a WTD is developed. The compressed dictionary is then used for sentence recognition in a continuous speech recognition system. Experimental results indicate a substantial improvement of the recognition rate after compression.
\end{abstract}

\section{Introduction}

Several continuous speech recognition systems currently being developed use context-dependent models which seek to capture the pronunciation variations resulting from phonetic context effects. It has been observed that with these models, large vocabulary speech recognition systems usually necessitates to compress, from some standard references, a word phonetic transcription dictionary (WTD) $[1,2,3,4]$. Such a dictionary generally gives a single transcription for a lexicon entry. Continuous speech recognition systems have shown satisfactory results when using dictionaries prepared in this way $[3,5,6,7]$. However, because of the large variations in the pronounciation of a given word $[8,9,10,11,12,13$,$] ,$ it is very difficult, when compressing a dictionary, to capture its most representative variant. Word transcriptions are first obtained from the context-dependent phonemic model CODEPHON-STM based on the automatically expending speed and context (AESC) approach developed in our laboratory $[11,4]$, and then compressed into a more compact form to balance the need between flexibility and reliability using an automatic procedure. In fact, the direct word transcriptions given by CODEPHON-STM can yield many transcriptions for a single lexicon entry. 
The different transcriptions of a word could reflect differences in dialect, phonetic contexts and speaking rates [11]. As it is well known, too many variations in transcribing words can also lead to an increased word confusions. In this paper, we describe a method to compress a multiple word transcription dictionary which gives good results. The method is based on a statistical modelling of the speech, and selects the transcriptions that maximise some likelihood function. The compressed dictionary is then used for sentence recognition in ML-vinics $[14,6,15,16]$, our multi-langual phoneme-based continuous speech recognizer. On a task of an 1070 word vocabulary and a test set grammar perplexity of 20 , the system achieves a word accuracy of $94.9 \%$, for a normal speed, which represents an error reduction of about $72 \%$ compared to the recognition results when using a standard dictionary.

This paper is organized into five sections. For providing back-ground material, the AESC approach and the phonemic model are briefly described in section 2. In section 3 , the algorithm for compressing the word transcription dictionaries is described. Experimental results on the Agrmmo database using the ML-VINICS system are provided in section 4 . Section 5 contains a conclusion.

\section{Preparing a WTD Using the AESC Approach}

\subsection{The AESC Approach}

The AESC purpose is to deduce context and speed dependent rules (CSDR) rules from a large labeled continuous speech corpus in order to construct a mapping between the symbolic phonemes in the WTD and their real pronunciations from the labeled data-base $[4,9,11,17,18,14]$. The AESC approach goes through the following steps:

- Step-1: training of the phonemic model

1. Labelling of a large continuous speech corpus

2. Segmentation of this speech corpus

3. Determination of its triplets

4. Identification of the phonemes in the middle of each triplet

5. Generation of the context and speed dependent rules (CSDR)

- Step-2: preparing the new WTD

1. Computation of the speaking rate

2. Loading of the CSDR corresponding to this speed

3. Tranformation of the sequence in the WTD of ML-VINICs with the loaded CSDR

4. Generation of the new WTD

\subsection{The CODEPHON-STM Model}

In this section we briefly examine a context-dependent phonemic model which uses a statistical method to generate the CDSRs. Furthers details are given in [4]. 
To model the contextual and speaking rate influence, we propose a stochastic trajectory model. A speech signal representing a symbol can be represented, in a parametric space, as a point which moves as articulatory configuration changes $[19,20,21]$. The trace of this moving point is called the trajectory of the symbol. Several techniques were applied to model these trajectories [22, 23, 24, 25].

Let us consider a sequence of parameter vectors: $\mathbf{v}_{0}, \mathbf{v}_{1}, \ldots \mathbf{v}_{t}, \ldots, \mathbf{v}_{T}$, where $T$ is the total number of vectors in a given utterance. Each point $\mathbf{v}_{t} \in \mathbb{R}^{D}$ is a $D$-dimensional vector in some parameter space. Let $\mathbf{V}_{t}$ be a subsequence of $L$ vectors centered at time slot $t$ :

$$
\mathbf{V}_{t} \triangleq \mathbf{v}_{t-\frac{L}{2}}, \mathbf{v}_{t-\frac{L}{2}+1}, \ldots \mathbf{v}_{t}, \ldots \mathbf{v}_{t-\frac{L}{2}+L-1}
$$

Let $\mathbb{P} H \triangleq\left\{p h_{1}, p h_{2}, \ldots, p h_{M}\right\}$ be a set of $M$ symbols representing phonemes. In our formulation, it is assumed that to each phoneme symbol are associated $N$ stochastic generators of trajectories, $T_{1}, T_{2}, \ldots, T_{N}$. Let $p f\left(\mathbf{V}_{t}, T_{n}, d, p h\right)$ be the joint probability density function (pdf) of vector sequence $\mathbf{V}_{t}$, trajectory source $T_{n}$, duration $d$ and phoneme symbol $p h \in \mathbb{P} H$.

$$
p f\left(\mathrm{~V}_{t}, T_{n}, d, p h\right)=p f\left(\mathrm{~V}_{t} \mid T_{n}, d, p h\right) P\left(T_{n} \mid d, p h\right) P(d \mid p h) P(p h)
$$

where

- $p f\left(\mathrm{~V}_{t} \mid T_{n}, d, p h\right)$ is the pdf of $\mathrm{V}_{t}$ given $T_{n}, d$ and $p h$,

- $P\left(T_{n} \mid d, p h\right)$ the probability of $T_{n}$ given $d$, and $p h$,

- $P(d \mid p h)$ the probability of $d$ given $p h$, and

- $P(p h)$ the a prior probability of $p h$.

We use $p f$ for continuous probability density functions and $P$ for discrete probabilities. The marginal pdf $p f\left(\mathrm{~V}_{t}, p h\right)$ can be obtained by summing up $p f\left(\mathrm{~V}_{t}, T_{n}, d, p h\right)$ over all trajectories $T_{n}$ and all durations $d$ :

$$
p f\left(\mathrm{~V}_{t}, p h\right)=P(p h) \sum_{d} \sum_{n=1}^{N} p f\left(\mathrm{~V}_{t} \mid T_{n}, d, p h\right) P\left(T_{n} \mid d, p h\right) P(d \mid p h)
$$

The probability of phoneme $p h$ given observation $\mathbf{V}_{t}$ is therefore:

$$
P\left(p h \mid \mathrm{V}_{t}\right)=\frac{p f\left(\mathrm{~V}_{t}, p h\right)}{p f\left(\mathbf{V}_{t}\right)}=\frac{P(p h)}{p f\left(\mathbf{V}_{t}\right)} \sum_{d} p f\left(\mathrm{~V}_{t} \mid d, p h\right) P(d \mid p h)
$$

where $p f\left(\mathbf{V}_{t} \mid d, p h\right)$ is the pdf of $\mathbf{V}_{t}$ given $d$ and $p h$ :

$$
p f\left(\mathbf{V}_{t} \mid d, p h\right) \triangleq \sum_{n=1}^{N} p f\left(\mathbf{V}_{t} \mid T_{n}, d, p h\right) P\left(T_{n} \mid d, p h\right)=\sum_{n=1}^{N} p f\left(\mathbf{V}_{t} \mid T_{n}, d, p h\right) P\left(T_{n} \mid p h\right)
$$

where we assume that for a given phoneme, the probability of a trajectory source $T_{n}$ does not depend on durations:

$$
P\left(T_{n} \mid d, p h\right)=P\left(T_{n} \mid p h\right)
$$


The duration propbability of each phoneme symbol $P(d \mid p h)$ is modeled by a $\Gamma$-distribution. The plausibility of phoneme is defined by Eq-4. Since $p f\left(\mathbf{V}_{t}\right)$ in this equation does not depend on $p h$, for our propose of phoneme recognition, i.e. finding the best $p h$, it is a constant and will be discarded. Also, $\sum_{d}$ in Eq-4 is changed to $\max _{d}$. Based on Eq-4 we define the plausibility of symbol $p h$ at time slot $t, \mu_{t, p h}$, as the logarithmic pdf of $p h$ given observation sequence $\mathbf{V}_{t}$ :

$$
\begin{gathered}
\mu_{t, p h} \triangleq \log P\left(p h \mid \mathrm{V}_{t}\right)=\log \left[P(p h) \max _{d}\left\{p f\left(\mathrm{~V}_{t} \mid d, p h\right) P(d \mid p h)\right\}\right] \\
0 \leq t<T, p h \in \mathbb{P} H .
\end{gathered}
$$

After carrying out this step for all the triplets, we get a set of rules for which the condition parts represent the possible triplets of the data base and the production parts represent the phoneme corresponding to the computed $\mu_{t, p h}$. For each speed, we call this set of rules CSRD. Using CSDR, we change the WTD [15] of ML-Vinics. As a result, we obtain, for each word in the lexicon, an important number of transcriptions. An automatic procedure is developed to compress the dictionary. The procedure is repeated for all the words in the recognition vocabulary. This newly obtained WTD is used to test the ML-VINICs system. The details of this compression procedure are given in the sequel.

\section{Dictionary Compression}

\subsection{Problem Formulation}

From sampled speech signal of an utterance, cepstral parameters are computed with $32 \mathrm{~ms}$ Hamming window each $10 \mathrm{~ms}$, resulting in a sequence of $N$ parameter vectors. Let $W$ be the number of words in the lexicon and $K$ the number of phonetic transcriptions of each word. Let $\mathbb{L} \triangleq\left\{p_{1}, p_{2}, \ldots, p_{L}\right\}$ be the set of $L$ phonetic symbols. We assume that a phonetic transcription of a word is a sequence of phonetic symbols $s_{n}$ :

$$
T \triangleq\left[s_{0}, s_{1}, \ldots s_{n}, \ldots s_{S(T)}\right], \forall n, s_{n} \in \mathbb{L}
$$

In the same manner we suppose that a word $m$ belonging to the lexicon may have several phonetic transcriptions:

$$
\alpha(m) \triangleq\left\{T_{1}, \ldots T_{k}, \ldots T_{K(m)}\right\}
$$

We denote by $\mu_{i, s}$ the likelihood of an utterance at time slot $i$, given the phonetic symbol $s \in \mathbb{L} . \mu_{i, s}$ can be computed with any adequate method, for example stochastic trajectory models [4]. The duration of a phone is considered as a random variable denoted $\rho$ and the probability for phone $s_{n}$ with duration $\rho=d$ is denoted $P\left(d \mid s_{n}\right)$. Let $t_{n}$ be the time index corresponding to the beginning of the sequence of vectors composing symbol $s_{n}$. We introduce the 
cumulative likelihood of $s_{n}$ as being the product of the likelihoods from $t_{n}$ to $t_{n+1}-1$, weighted by the duration probability:

$$
Q\left(s_{n}\right) \triangleq P\left(t_{n+1}-t_{n} \mid s_{n}\right)^{\varepsilon} \prod_{t_{n} \leq i<t_{n+1}} \mu_{i, s_{n}}
$$

where $\varepsilon \geq 0$ is a constant. We then introduce the cumulative likelihood of a transcription, i.e., a sequence of phonetic symbols:

$$
\phi\left(T \mid t_{0}, t_{1}, t_{2}, \ldots t_{S(T)}\right)=\frac{1}{N} \prod_{s \in T} Q(s)
$$

with $t_{0}=0, t_{n}<t_{n+1}$, and $t_{S(T)}=N-1$, where $N$ is the total number of vectors in the utterance. Therefore, this plausibility is a function of $t_{n}, \forall n \in[0, S(T)-1]$. By optimising the $t_{n}$, the value of $\phi(T)$ can be maximised in the following manner:

$$
\Phi(T) \triangleq \max _{t_{1}, \ldots t_{S(T)}} \phi\left(T \mid t_{1}, \ldots t_{S(T)}\right)
$$

For a given word $m$, the application of equation 12 to each of its transcriptions $T_{k} \in \alpha(m)$ results in a particular value $\Phi\left(T_{k}\right)$. We denote by $\operatorname{argmax}_{T \in T}(\Phi(T), j)$ the function that returns the transcription that gives the $j^{\text {th }}$ largest value of $\Phi$ given the transcription set $\mathcal{T}$. The optimum subset of $M$ transcriptions of the word $m$ is then given by:

$$
\hat{\alpha}(m) \triangleq\left[\hat{T}_{1}, \hat{T}_{2}, \ldots, \hat{T}_{M}\right]_{m}
$$

Where

$$
\hat{T}_{j} \triangleq \underset{T \in \alpha(m)}{\operatorname{argmax}}(\Phi(T), j)
$$

Only these $M$-best transcriptions are kept in the sequel at the level of recognition. All other transcriptions are discarded.

\subsection{Implementation}

Direct application of equation 14 requires precise specification of the beginning and ending positions of a word in the training utterance, which may change as a function of the transcriptions and thus cannot be determined reliably. To avoid such a specification, we need to generate phonetic transcriptions at the utterance level. For a given word $m$, the generator performs three steps:

- An utterance baseline transcription using a single transcription word dictionary is built by simply concatenating the transcriptions of the words composing an utterance.

- The transcription of the word $m$ is replaced by each of the $K(m)$ transcriptions, yielding $K(m)$ utterance transcriptions where transcriptions for words other than $m$ remain unchanged.

- These utterance transcriptions are used by equation 14 to find out the $M$-best transcriptions of the word.

The procedure is repeated for all words in the recognition vocabulary. 


\section{Experiments}

We used, for the training step of the phonemic model (step-1), a first labeled corpus (corpus- $I$ ) consisting of 1780 sentences pronounced three times (with different speeds) by a male speaker. For the continuous speech recognition, we have collected from a regional newspaper a text corpus (AGIMMo) consisting of real estate advertisements. The recognition task of the corpus is described by a grammar [15] of 11030 rules with a vocabulary of 1070 words and a bigram perplexity of 20. The grammar was compiled with our phonetic model CoDEPHON-STM into a non-deterministic automaton of about 903619 states, in which transition labels are phoneme symbols. The speech database consists of 1200 sentences pronouced by eight male and two female French speakers. Each speaker read 300 sentences from the corpus. 140 sentences per speaker were used to train the acoustic models of the recognition system, and the remaining 160 were used for testing. Before compression, the CODEPHON-STM model using the AESC approach generated for each word an average of 30 phonetic transcriptions. The compression produced the 2 -best transcriptions for each word ( $M=2$ ).

\begin{tabular}{|l|c|c|c|c|c|c|c|c|c|}
\hline \multirow{2}{*}{ SPEAKER } & \multicolumn{3}{|c|}{ SPEED-1 (NORMAL) } & \multicolumn{3}{c|}{ SPEED-2 (LOW) } & \multicolumn{3}{c|}{ SPEED-3 (HIGH) } \\
\cline { 2 - 10 } & $\mathrm{S}$ & $\mathrm{E}(30)$ & $\mathrm{C}(2)$ & $\mathrm{S}$ & $\mathrm{E}(30)$ & $\mathrm{C}(2)$ & $\mathrm{S}$ & $\mathrm{E}(30)$ & $\mathrm{C}(2)$ \\
\hline \hline brs (F) & 75.3 & 87.8 & 92.3 & 76.9 & 88.1 & 87.8 & 76.3 & 87.2 & 88.8 \\
\hline crm & 75.8 & 88.1 & 92.8 & 68.8 & 84.1 & 88.1 & 67.5 & 78.6 & 84.4 \\
\hline jel & 61.4 & 76.5 & 88.7 & 55.0 & 72.8 & 76.5 & 54.1 & 70.8 & 76.7 \\
\hline lar & 93.1 & 95.6 & 97.8 & 83.1 & 92.8 & 95.6 & 82.7 & 90.1 & 94.0 \\
\hline ols & 93.2 & 98.0 & 98.9 & 85.0 & 92.5 & 98.0 & 83.1 & 93.8 & 98.4 \\
\hline sat & 75.8 & 85.9 & 96.1 & 75.0 & 86.4 & 85.9 & 62.5 & 81.0 & 86.1 \\
\hline std & 86.3 & 89.2 & 93.9 & 79.4 & 92.5 & 89.2 & 74.4 & 88.0 & 92.8 \\
\hline syc (F) & 71.8 & 81.9 & 89.9 & 68.1 & 81.0 & 82.0 & 71.9 & 84.3 & 81.0 \\
\hline vil & 88.7 & 93.8 & 98.4 & 81.9 & 89.5 & 93.8 & 81.9 & 89.5 & 90.4 \\
\hline yig & 92.5 & 94.5 & 98.5 & 85.6 & 95.7 & 94.5 & 85.6 & 95.7 & 93.4 \\
\hline \hline AVERAGE & 81.4 & 89.2 & 94.9 & 75.9 & 87.6 & 89.2 & 74.0 & 86.0 & 88.6 \\
\hline
\end{tabular}

Table 1: Word recognition for standard (S), extended (E) and compressed (C) dictionaries for different speeds. The figures in parentheses show the number of transcriptions per word used.

In table 1, we report the word recognition rates for the standard single transcription dictionary, the extended dictionary and the compressed dictionary for different speeds. From this table, we see that by using the extended transcription dictionary instead of the single transcription one, the recognition is improved substantially for every speakers. On the average, the recognition percentage increased from $81.4 \%$ to $89.2 \%$, yielding an error rate reduction of about $50 \%$. The compression improved consistently the recognition percentage for all speakers and further reduced the remaining error by about $22 \%$. It is worth noticing that the complexity of the recognition task increases exponentially with the num- 
ber of transcriptions per word. Therefore in reducing the average trandcription number from 30 to 2 , we saved in computation time and gained a significant memory space.

\section{Conclusion}

In this paper we have investigated the use of a method for preparing a WTD in the hope of obtaining a better decoding accuracy than with word transcriptions in the forms of isolated utterances. WTDs are first obtained from a contextdependent phonemic model based on the AESC approach, and then compressed using a statistical cost measure of some likelihood function. Both the compressed, the extended and the standard dictionary are used in evaluating the decoding performance of an automatic continuous speech recognition system. Experimental results indicate a substantial improvement of the recognition rate after compression. Moreover, the compression procedure seems to be capable of capturing the most representative pronunciations from among many variations. In the other hand, since the between-word context dependence has not been incorporated into the context-dependent phonemic model at this stage of decoding, the advantage of using multiple transcriptions might not have been fully utilized.

\section{REFERENCES}

[1] Y. Zhao. A speaker-independent continuous speech recognition system using continuous mixture gaussian density HMM of phoneme-sized units. IEEE Trans. on Speech and Audio Processing, 1(3):345-361, July 1993.

[2] L. R. Bahl, P. F. Brown, P. V. de Souza, R. L. Mercer, and M. A. Picheny. A method for the construction of acoustic Markov models for words. IEEE Trans. on Speech and Audio Processing, 1(4):443-452, October 1993.

[3] Y. Zhao, H. Wakita, and X. Zhuang. Generate word transcription dictionary from sentence utterances and evaluate its effects on speaker independant continuous speech recognition. In Proceedings of European Conference on Speech Technology, pages 679-682, Genova, Italy, September 1991.

[4] F. Mouria-Beji. Context and Speed Dependent Phonetic Models for Continuous Speech Recognition. In ESCA Tutorial Proceedings of Modeling Pronunciation Variation for Automatic Speech Recognition, Kerkrade, Netherlands, May 1998.

[5] K.F. Lee. Large-vocabulary speaker-independent continuous speech recognition: the SPHINX system. PhD thesis, Carnegie Mellon Univ., Pittsburgh, PA, April 1988.

[6] F. Beji Mouria. Un Système de Reconnaissance de la Parole Continue et son Expérimentation avec un Large Vocabulaire. To appear in revue magrébine de l'ingénieur. 1998.

[7] C.H. Lee. Acoustic modeling of subword units for speech recognition. In Proceedings of IEEE International Conference on Acoustics, Speech and Signal Processing, pages 721-724, Albuquerque, USA, April 1990.

[8] L.R. Bahl, P.V. de Souza, P.S. Gopalakrishnan, N. Nahamoo, and M.A. Pichney. Decision trees for phonological rules in continuous speech. In Proceedings of IEEE International Conference on Acoustics, Speceh and Signal Processing, pages 185-188, Toronto, Canada, May 1991.

[9] F. Mouria-Beji. CODEPHON-NN: A COntext-DEpendent PHONemic model based on Neural Networks. In Computational Engineering in Systems Applicatians multiconference, CESA'98. IEEE-SMC, April 1998. 
[10] R.M. Schwartz, Y. Chow, O.A. Kimball, S. Roucos, M. Krasner, and J. Makhoul. Contextdependent modeling for acoustic-phonetic recognition of continuous speech. In Proceedings of IEEE International Conference on Acoustics, Speech and Signal Processing, pages 1205-1208, Tampa, Florida, March 1985.

[11] F. Mouria-Beji, Y. Gong, and J. P. Haton. Use of explicit context-dependent phonemic model in contimuous speech recognition. In Proc. European Conf. on Speech Communication and Technology, pages 2223-2226, Berlin, Germany, 1993,EUROSPEECH'93.

[12] L.R. Bahl, P.V. de Souza, P.S. Gopalakrishnan, D. Nahamoo, and M. Picheney. Word lookahead scheme for cross-word right context models in a stack decoder. In Proceedings of European Conference on Speech Technology, pages 851-854, Berlin, 1993.

[13] K.F. Lee. Context dependent phonetic hidden Markov models for speaker independent continuous speech recognition. IEEE Trans. on Acoust., Speech and Signal Processing, 38(4):599-609, April 1990.

[14] F. Mouria-Beji. A Multi-Lingual Continuous Speech Recognition System. In Proc. 6th International Conference and Exhibition on Multi-lingual Computing, Cambridge, UK, April 1998. ICEMCO-98.

[15] Y. Gong, J.-P. Haton, and F. Mouria-Beji. Continuous speech recognition based on high plausibility regions. In Proc. IEEE Int. Conf. on Acoustics, Speech and Signal Processing 1991, volume 1, pages 725-728, Toronto, Canada, May 1991, IEEE-ICASSP'91.

[16] F. Mouria-Beji. Neural network use in a non-linear vectorial interpolation technique for speaker recognition. In IEEE World Congress on Computational Intelligence, Anchorage, Alaska, May 1998,IEEE WCCI.

[17] F. Mouria-Beji, Y. Gong, and J. P. Haton. Un modèle phonétique tenant compte explicitement du contexte pour la reconnaissance de la parole. In Actes du $9^{\text {ème }}$ congrès Reconnaissance des Formes et Intelligence Artificielle, volume 1, pages 265-275, Paris, France, January 1994, AFCET RFIA'94.

[18] F. Mouria-Beji and J. P. Haton. Utilisation des réseaux de neurones pour le traitement de la variabilité du signal de parole. In The $15^{\text {th }}$ Tünsian Conference on Electrical Machinery and Automatic Controle, volume 1, pages 122-130, Nabeul, November. 1995. JTEA'95,

[19] S. Takahashi, T. Matsuoka, Y. Minami, and K. Shikano. Phoneme HMMS constrained by frame correlations. In Proceedings of International Conference on Acoustics, Speech and Signal Processing, volume II, pages 219-222, 1993.

[20] M. Ostendorf and S. Roucos. A stochastic segment model for phoneme-based continuous speech recognition. IEEE Trans. Acoust., Speech and Signal Processing, 37(12):1857$1869,1989$.

[21] V. V. Digalakis, M. Ostendorf, and J. R. Rohlicek. Fast algorithms for phone classification and recognition using segment-based models. IEEE Trans. on Signal Processing, 40(12):2885-2896, Dec. 1992.

[22] S. Furui. Speaker-independent isolated word recognition using dynamic features of speech spectrum. IEEE Trans. Acoust., Speech and Signal Processing, ASSP-34(1):53-59, 1986.

[23] Wellekens. Explicite time correlation in hidden Markov models for speech recognition. In Procedings of International Conference on Acoustics, Speech and Signal Processing, pages 384-386, Dallas, 1987.

[24] K. K. Paliwal. Use of temporal correlation between successive frames in a hidden Markov model based speech recognizer. In Proceedings of International Conference on Acoustics, Speech and Signal Processing, volume II, pages 215-218, 1993.

[25] T. Robinson. A real-time recurrent error propagation network word recognition system. In Proceedings of International Conference on Acoustics, Speech and Signal Processing, volume I, pages $617-620,1992$. 